\title{
A NOTE ON INITIAL SEGMENTS OF THE ENUMERATION DEGREES
}

\author{
THEODORE A. SLAMAN AND ANDREA SORBI
}

\begin{abstract}
We show that no nontrivial principal ideal of the enumeration degrees is linearly ordered: In fact, below every nonzero enumeration degree one can embed every countable partial order. The result can be relativized above any total degree: If $\mathbf{a}, \mathbf{b}$ are enumeration degrees, with $\mathbf{a}$ total, and $\mathbf{a}<\mathbf{b}$, then in the degree interval $(\mathbf{a}, \mathbf{b})$, one can embed every countable partial order.
\end{abstract}

\section{INTRODUCTION}

A distinguishing feature of enumeration reducibility (for short, e-reducibility) compared to the main computability theoretic reducibilities, including Turing reducibility, is downwards density, i.e., the nonexistence of minimal elements in the poset of the corresponding degree structure. Whether or not there exists a minimal enumeration degree (for short, e-degree) was in fact an open problem for some years, raised by Rogers [12, p. 282], and finally answered in the negative by Gutteridge [5]. A printed presentation of Gutteridge's result can be found in [2], or in $[10$, p. 837].

Apart from downwards density, very little is known about the initial segments of the e-degrees, not even if there are linearly ordered nontrivial initial segments. In this note we answer this question, by showing that downwards density can always be accompanied by incomparability. Indeed the following holds, where $\mathbf{0}_{e}$ denotes the least e-degree:

Theorem 1.1. For every e-degree $\mathbf{b}>\mathbf{0}_{e}$ the interval $\left(\mathbf{0}_{e}, \mathbf{b}\right)$ contains incomparable degrees, in fact one can embed every countable partial order in it.

The previous theorem can be partially relativized as follows (recall that an edegree is total if it contains the graph of a total function):

Theorem 1.2. If $\mathbf{a}, \mathbf{b}$ are e-degrees, with $\mathbf{a}$ total, and $\mathbf{a}<\mathbf{b}$, then in the degree interval $(\mathbf{a}, \mathbf{b})$, one can embed every countable partial order.

In fact Theorem 1.1 is a particular case of Theorem 1.2, as $\mathbf{0}_{e}$ is total.

2010 Mathematics Subject Classification. 03D30.

Key words and phrases. Enumeration reducibility, enumeration degree.

This research was carried out while the second author was visiting the Department of Mathematics of the University of California, Berkeley, during April and May 2011. The authors would like to thank the Isaac Newton Institute for Mathematical Sciences of Cambridge, England, for its hospitality during the final phase this paper was completed. The first author was partially supported by NSF award DMS-1001551. The second author was partially supported by the project Computability with partial information, sponsored by BNSF, Contract No: D002-258/18.12.08. 
Our notations and terminology regarding computability theory are standard, and can be found for instance in [4], or [15]; for a thorough introduction to the enumeration degrees, the reader is referred to [3], or [10, Chapter XIV]. Each set of numbers $\Theta$ can be viewed as a generalized enumeration operator, mappings sets to sets: We write $\Theta^{A}$ to denote the image of the set $A$ under $\Theta$,

$$
\Theta^{A}=\{x:(\exists \text { finite } D)[\langle x, D\rangle \in \Theta \text { and } D \subseteq A]\},
$$

where finite sets are identified with their canonical indices. If $\Theta$ is a computably enumerable set, then the associated mapping is called an enumeration operator (for short, e-operator). Let $\left\{W_{e}: e \in \omega\right\}$ be the standard numbering of the computably enumerable sets, together with suitable computable approximations $\left\{W_{e}^{s}: e, s \in \omega\right\}$ consisting of finite sets: As usual, we denote by $\Phi_{e}$ and $\Phi_{e}^{s}$ the e-operators defined by $W_{e}$ and $W_{e}^{s}$, respectively. We use $\leq_{e}$ to denote $e$-reducibility, i.e., $A \leq_{e} B$ if $A=\Phi^{B}$ for some e-operator $\Phi$; we denote by $\operatorname{deg}_{e}(A)$ the $e$-degree of $A$, i.e., the equivalence class of $A$ under the equivalence relation $\equiv_{e}$ induced by $\leq_{e}$. We say that two sets $A$ and $B$ are e-incomparable if neither $A \leq_{e} B$ nor $B \leq_{e} A$. Finally, we use the notation $\omega^{[i]}=\{\langle i, x\rangle: x \in \omega\}$, where $\langle.,$.$\rangle is the Cantor pairing function.$

\section{The Main Lemma}

In this section we present the main combinatorial argument on which Theorem 1.1 and Theorem 1.2 are based, i.e., we show (Lemma 2.3, called the Main Lemma) how to construct a computably independent collection of e-degrees, uniformly below a given e-degree not containing any $\Delta_{2}^{0}$ set, which, together with Lemma 2.2, immediately yields Theorem 1.1. In Section 3 we will then indicate how to modify the proof of Lemma 2.3 to get Theorem 1.2.

A countable set of e-degrees $\left\{\mathbf{a}_{i}: i \in \omega\right\}$ is computably independent, if for every $i \in \omega$, we have that

$$
\mathbf{a}_{i} \not \bigoplus_{r \neq i} \mathbf{a}_{r}
$$

where, for every family $\left\{A_{r}: r \in R\right\}$ of sets, with $R \subseteq \omega$, we set

$$
\bigoplus_{r \in R} A_{r}=\bigcup_{r \in R}\{r\} \times A_{r}, \quad \text { and } \bigoplus_{r \in R} \operatorname{deg}_{e}\left(A_{r}\right)=\operatorname{deg}_{e}\left(\bigoplus_{r \in R} A_{r}\right) \text {. }
$$

A countable collection $\left\{\mathbf{a}_{i}: i \in \omega\right\}$ of e-degrees is said to be uniformly below $\mathbf{y}$, for a given e-degree $\mathbf{y}$, if $\bigoplus_{r \in \omega} \mathbf{a}_{r} \leq_{e} \mathbf{y}$.

Lemma 2.1. If $(\mathbf{x}, \mathbf{y})$ is an interval of e-degrees which contains a computably independent set of e-degrees, which is uniformly below $\mathbf{y}$, then every countable partial order can be embedded in the interval.

Proof. The proof of this follows the pattern of the proof of the analogous result for the Turing degrees, due to Sacks [13]: See for instance [8] for an adaptation of this proof to the e-degrees. Uniformity is used to guarantee that the range of the constructed embedding is below $\mathbf{y}$.

We recall:

Lemma 2.2 (Lagemann [7]). If $\mathbf{b}>\mathbf{0}_{e}$, and $\mathbf{b}$ contains a $\Delta_{2}^{0}$ set, then below $\mathbf{b}$ one can embed every countable partial order. 
Proof. See [7]. A clear exposition of the proof can be found also in [9]. It is worth mentioning that under the assumption, one can always construct an embedding with range included in the $\Delta_{2}^{0}$ e-degrees.

We are now ready to prove the main result of this paper:

Lemma 2.3 (Main Lemma). There exists a collection of e-operators $\left\{\Theta_{r}: r \in \omega\right\}$ such that, for every set $B$, the set of e-degrees $\left\{\operatorname{deg}_{e}\left(\Theta_{r}^{B}\right): r \in \omega\right\}$ is uniformly below $\operatorname{deg}_{e}(B)$, and if $B \notin \Delta_{2}^{0}$ then $\left\{\operatorname{deg}_{e}\left(\Theta_{r}^{B}\right): r \in \omega\right\}$ is computably independent.

As a corollary, we have:

Corollary 2.4. Every countable partial order can be embedded below any e-degree $\mathbf{b}>\mathbf{0}_{e}$. In particular, below any e-degree $\mathbf{b}>\mathbf{0}_{e}$ there exist incomparable e-degrees.

Proof. Let $\mathbf{b}>\mathbf{0}_{e}$, and let $B \in \mathbf{b}$. Let $\left\{\Theta_{r}: r \in \omega\right\}$ be the e-operators given by the Main Lemma. Either the set $\left\{\operatorname{deg}_{e}\left(\Theta_{r}^{B}\right): r \in \omega\right\}$ is computably independent and then we can apply Lemma 2.1 relative to the interval $\left(\mathbf{0}_{e}, \mathbf{b}\right)$, or $B \in \Delta_{2}^{0}$, but in this case the result is guaranteed by Lemma 2.2.

Proof of Main Lemma. We use a variation on Gutteridge's basic ideas, that are behind his proof of the nonexistence of a minimal e-degree, to build a computably enumerable class of e-operators $\left\{\Theta_{r}: r \in \omega\right\}$ (i.e., the set $\left\{\langle x, r\rangle: x \in \Theta_{r}\right\}$ is computably enumerable) such that, for every $B$, if $\left\{\operatorname{deg}_{e}\left(\Theta_{r}^{B}\right): r \in \omega\right\}$ is not computably independent, then $B \in \Delta_{2}^{0}$. Recall that Gutteridge builds an e-operator $\Theta$ such that, for every set $B$ :

- If $\Theta^{B}$ is computably enumerable, then $B \in \Delta_{2}^{0}$;

- if $B \leq_{e} \Theta^{B}$ then $B$ is computably enumerable:

The nonexistence of minimal e-degrees follows then from the fact that if $B$ is not computably enumerable then either $B$ is $\Delta_{2}^{0}$, in which case the e-degree of $B$ can not be minimal by Lemma 2.2 (Lagemann's proof relies on a previous result by Gutteridge [5] showing that every nonzero $\Delta_{2}^{0}$ e-degree properly bounds a nonzero e-degree); or $\emptyset<_{e} \Theta^{B}<_{e} B$.

As our e-operators form a computably enumerable class, it is also easy to see that for every $B$, the set $\left\{\operatorname{deg}_{e}\left(\Theta_{r}^{B}\right): r \in \omega\right\}$ is uniformly below $\operatorname{deg}_{e}(B)$.

The requirements. We want to build a computably enumerable class of e-operators $\left\{\Theta_{r}: r \in \omega\right\}$ such that the following requirements are satisfied, for every $i, r \in \omega$ :

$$
R_{i}^{r}: \quad\left(\forall B \notin \Delta_{2}^{0}\right)\left[\Theta_{r}^{B} \neq \Phi_{i}^{\oplus_{j \neq r} \Theta_{j}^{B}}\right] .
$$

We will ensure that for every $j, \Theta_{j}^{\omega} \subseteq \omega^{[j]}$, thus, for every set $B$,

$$
\bigoplus_{j \neq r} \Theta_{j}^{B} \equiv_{e}\left(\bigcup_{j \neq r} \Theta_{j}\right)^{B},
$$

so that, writing $\Theta_{\neq r}=\bigcup_{j \neq r} \Theta_{j}$, we may assume that the requirement $R_{i}^{r}$ has in fact the form $\left(\forall B \notin \Delta_{2}^{0}\right)\left[\Theta_{r}^{B} \neq \Phi_{i}^{\Theta_{\neq r}^{B}}\right]$. (If $\Theta_{r}^{B} \neq \Phi_{i}^{\oplus_{j \neq r} \Theta_{j}^{B}}$ then we say that $B$ satisfies $R_{i}^{r}$.)

We also fix a computable linear ordering $\leq$ of the requirements, with least requirement $R_{0}^{0}$. 
Notations and terminology for binary strings. Let $2^{<\omega}$ be the full binary tree. The symbol $\lambda$ denotes the empty string; $|\sigma|$ denotes the length of $\sigma$, and we define

$$
\sigma^{=1}=\{i<|\sigma|: \sigma(i)=1\} .
$$

If $\tau$ is a prefix of $\sigma$ then we write $\tau \subseteq \sigma$, with $\tau \subset \sigma$ meaning that $\tau \subseteq \sigma$ but $\tau \neq \sigma$. We write $\sigma<\tau$ to denote that either $|\sigma|<|\tau|$, or $|\sigma|=|\tau|$ and $\sigma$ precedes $\tau$ in the reverse lexicographical order (i.e., $\sigma \neq \tau$ and on the least bit $i$ on which $\sigma$ and $\tau$ differ, we have that $\sigma(i)=1$ and $\tau(i)=0$ ); finally, $\sigma \leq \tau$ stands for $\sigma<\tau$ or $\sigma=\tau$. Notice that $<$ is a linear ordering on strings. We say that $\sigma$ has higher priority than $\tau$ if $\sigma<\tau$.

Given a set $B$, we use the same symbol $B$ to denote both the set and its characteristic function, relying on the context to clarify which one is which: For instance, if we write $\sigma^{=1} \subseteq B$ or $|\sigma| \in B$ then we mean the set, whereas if we write $\sigma \subset B$ then we mean the characteristic function of the set (specifically, $\sigma \subset B$ means that the string $\sigma$ is a prefix of the characteristic function of $B$ ).

Given strings $\sigma$ and $\tau$, the symbol $\widehat{\sigma^{\wedge} \tau}$ denotes the concatenation of the two strings. For $\sigma \neq \lambda$, let $\sigma^{-}$denote the unique string $\sigma^{-} \subset \sigma$ such that $|\sigma|=\left|\sigma^{-}\right|+1$. If $\sigma \subset B$, then let $\sigma^{+, B}$ denote the string immediately following $\sigma$ along $B$, i.e., $\sigma^{+, B}=\sigma^{\wedge}\langle B(|\sigma|)\rangle$, or, equivalently, $\sigma^{+, B}=B \uparrow(|\sigma|+1)$.

Brief description of the strategies, and their environments. The construction is by stages. At stage $s$, for every $r \in \omega$, we define a finite approximation $\Theta_{r}^{s}$ to the e-operator $\Theta_{r}$ (such that the predicate " $x \in \Theta_{r}^{s}$ ", in $x, r, s$, is computable, thus guaranteeing that the constructed family of e-operators forms a computably enumerable class), and for every $\sigma \in 2^{<\omega}$, we specify the current states of several parameters relative to $\sigma$. In detail:

We set the value $R(\sigma, s)$ of a requirement assigned to $\sigma$, such that eventually, $\lim _{s} R(\sigma, s)=R(\sigma)$ exists. If $R(\sigma, s)=R_{i}^{r}$, we let $r(\sigma, s)=r, \Theta_{\sigma}^{s}=\Theta_{r}^{s}, \Theta_{\neq \sigma}^{s}=$ $\Theta_{\neq r}^{s}$, and $\Phi_{\sigma}^{s}=\Phi_{i}^{s}$. Similarly, if $R(\sigma)=R_{i}^{r}$ then $r(\sigma)=\lim _{s} r(\sigma, s)=r$, and we write $\Theta_{\sigma}=\Theta_{r}, \Theta_{\neq \sigma}=\Theta_{\neq r}$, and $\Phi_{\sigma}=\Phi_{i}$.

Finally, we set also the value of a parameter $j(\sigma, s)$, for which eventually we have that $\lim _{s} j(\sigma, s)=j(\sigma)$ exists, the limit being reached at the same stage as for $R(\sigma)$. The parameter $j(\sigma, s)$ will be chosen from the set $\omega^{[r(\sigma, s)]}$.

Each $\sigma \in 2^{<\omega}$ can be regarded as a strategy working towards satisfaction of the currently assigned requirement $R(\sigma)$. The strategy for $\sigma$ can be roughly described as follows:

(1) appoint a witness $j(\sigma)$ and add the validating axiom $\left\langle j(\sigma), \sigma^{=1} \cup\{|\sigma|\}\right\rangle \in$ $\Theta_{\sigma}$

(2) wait for an opportunity of restraining $j(\sigma) \in \Phi_{\sigma}^{\Theta} \Theta_{\neq \sigma}^{B}$, any $B \supset \sigma$, by restraining $j(\sigma) \in \Phi_{\sigma}^{\Theta_{\neq}^{\sigma=1}}$ without interfering with higher priority strategies $\tau<\sigma$ : This can be done since, due to the form of the validating axioms, $\sigma$ has a good guess on which $j(\tau)$, with $\tau<\sigma$, are in $\Theta_{\tau}^{B}$, and hence in $\Theta_{\neq \sigma}^{B}$. If this restraining opportunity arises (say in this case that $j(\sigma)$ becomes realizable), then dump (possibly injuring lower priority strategies), i.e., permanently restrain in $\Theta_{\neq \sigma}$ using axioms of the form $\langle x, \emptyset\rangle \in \Theta_{r}$, all other elements needed to hold $j(\sigma) \in \Phi_{\sigma}^{\Theta_{\neq \sigma}^{B}}$ (after this action, we say that $j(\sigma)$ becomes realized); 
(3) notice, if $j(\sigma)$ becomes realized then any $B \supset \sigma$, with $|\sigma| \notin B$, satisfies the currently assigned $R(\sigma)$ : Define in this case $R\left(\sigma^{\wedge} 0\right)$ to be the next requirement to be satisfied, and repeat $R\left(\sigma^{\wedge} 1\right)=R(\sigma)$. Dually, if $j(\sigma)$ never becomes realized then any $B \supset \sigma$, with $|\sigma| \in B$, satisfies the currently assigned $R(\sigma)$ : Define in this case $R(\widehat{\sigma} 1)$ to be the next requirement to be satisfied, and repeat $R\left(\sigma^{\wedge} 0\right)=R(\sigma)$.

The above actions are of course framed in a (finite) priority argument. For every $\sigma$, the limit values $R(\sigma)$ and $j(\sigma)$ can be recovered in a $\Delta_{2}^{0}$ fashion: For these limit values, along any set $B$, we have that $R(\tau) \leq R(\sigma)$ for $\tau \subseteq \sigma$, and $R(\tau) \neq R\left(\tau^{+, B}\right)$ implies that $R\left(\tau^{+, B}\right)$ is the requirement immediately following $R(\tau)$ in the order of the requirements. Thus either all requirements are assigned to strings along $B$ (giving that $B$ satisfies all requirements, as $R(\tau) \neq R\left(\tau^{+, B}\right)$ means that $B$ satisfies $R(\tau)$ ); or the sequence of requirements eventually becomes constant along $B$ : In this case, since we can decide in a $\Delta_{2}^{0}$ fashion whether or not $j(\sigma)$ becomes eventually realized, for each sufficiently long given $\sigma$ we can also decide $B(|\sigma|)$ in a $\Delta_{2}^{0}$ fashion, as $B(|\sigma|)$ is the value that makes $B$ not to satisfy $R(\sigma)$, namely $B(|\sigma|)=1$ if and only if $j(\sigma)$ gets eventually realized.

The construction. We now present the formal construction. At stage $s+1$ any parameter retains the same value as at $s$, unless otherwise specified.

Definition 2.5. We say that a finite set $F$ is $\sigma$-accessible at $s+1$, if $F \cap \omega^{[r(\sigma, s+1)]}=$ $\emptyset$, and

$$
F \cap\{j(\tau, s+1): \tau \leq \sigma\} \subseteq\left\{j(\tau, s+1):|\tau|<|\sigma| \text { and } \tau^{=1} \cup\{|\tau|\} \subseteq \sigma^{=1}\right\} .
$$

We say that $j(\sigma, s+1)$ is realizable at $s+1$ if there exists a $\sigma$-accessible finite set $F$, such that $j(\sigma, s+1) \in \Phi_{\sigma}^{s F}$.

Definition 2.6. We initialize $\sigma$ at stage $s$, by setting $j(\sigma, s)$ and $R(\sigma, s)$ to be undefined. At stage $s+1$ we say that $\sigma$ is initialized, if $j(\sigma, s)$ and $R(\sigma, s)$ are undefined.

Stage 0. Let $R(\lambda, 0)=R_{0}^{0}$; pick $j(\lambda) \in \omega^{[0]}$, and let $j(\lambda, 0)=j(\lambda)$; let $\Theta_{0}^{0}=$ $\{\langle j(\lambda),\{0\}\rangle\}$, and $\Theta_{r}^{0}=\emptyset$ for all $r \neq 0$; initialize all $\sigma \neq \lambda$. (Notice, $\lambda$ will never be initialized, so, for every $s, j(\lambda, s)=j(\lambda)$, and $R(\lambda, s)=R_{0}^{0}$.)

Stage $s+1$. Pick the <-least string $\sigma$ which is initialized, or $j(\sigma, s+1)$ becomes realizable at $s+1$, but is not yet realized:

(1) If $\sigma$ is initialized, then define $R(\sigma, s+1)=R$ where

(a) $R$ is the least requirement such that $R \notin\{R(\tau, s+1): \tau \subset \sigma\}$, if either $j\left(\sigma^{-}, s+1\right)$ is not realized and $\sigma\left(\left|\sigma^{-}\right|\right)=1$, or $j\left(\sigma^{-}, s+1\right)$ is realized and $\sigma\left(\left|\sigma^{-}\right|\right)=0$;

(b) $R=R\left(\sigma^{-}, s+1\right)$, otherwise;

define $j(\sigma, s+1)$ to be a new number (i.e., bigger than the numbers so far mentioned in the construction) in $\omega^{[r(\sigma, s+1)]}$; add the validating axiom

$$
\left\langle j(\sigma, s+1), \sigma^{=1} \cup\{|\sigma|\}\right\rangle \in \Theta_{\sigma}^{s+1} ;
$$

(2) otherwise, i.e., $\sigma$ is not initialized, and $j(\sigma, s)$ becomes realizable at $s+1$, and $F$ is a corresponding least $\sigma$-accessible finite set, then for every $x$ with

$$
x \in F-\{j(\tau, s+1): \tau \leq \sigma\},
$$


$\sigma$-dump $x$ into $\Theta_{\neq \sigma}$, i.e., add the axiom $\langle x, \emptyset\rangle \in \Theta_{r}^{s+1}$, if $x \in \omega^{[r]}$. We say in this case that $j(\sigma, s)$ gets realized at $s+1$.

In either case, we say that $\sigma$ acts at $s+1$.

Go to stage $s+2$, and initialize all strings $\tau$, with $\tau>\sigma$. (Notice that at each stage there always is a string that acts.)

This ends the construction. For $r \in \omega$, let $\Theta_{r}=\bigcup_{s} \Theta_{r}^{s}$.

Proof that the construction works. The class $\left\{\Theta_{r}: r \in \omega\right\}$ is clearly computably enumerable, since the construction is computable: Recall that the approximating sequence $\left\{\Phi_{i}^{s}: i, s \in \omega\right\}$ is a computable sequence of finite sets, and looking for a suitable $\sigma$-accessible set at stage $s+1$ requires to check the finitely many axioms $\langle j(\sigma, s), F\rangle \in \Phi_{\sigma}^{s}$; hence one can effectively decide at $s+1$ whether $j(\sigma, s+1)$ is realizable, or already realized.

The rest of the proof breaks down into the following claims.

Claim 1. For every $\sigma, \lim _{s} j(\sigma, s)=j(\sigma)$ and $\lim _{s} R(\sigma, s)=R(\sigma)$ exist (and thus $\lim _{s} r(\sigma, s)=r(\sigma)$ exists as well). Moreover, for every $\sigma_{1}, \sigma_{2}$,

$$
\sigma_{1} \subseteq \sigma_{2} \Rightarrow R\left(\sigma_{1}\right) \leq R\left(\sigma_{2}\right),
$$

and if $R(\sigma) \neq R\left(\sigma^{-}\right)$then $R(\sigma)$ immediately follows $R\left(\sigma^{-}\right)$in the ordering $\leq$of the requirements.

Proof. The proof that $\lim _{s} j(\sigma, s)$ and $\lim _{s} R(\sigma, s)$ exist is by induction on the position $n$ of $\sigma$ in the order < of strings. For $n=0$, notice that, for every $s$, $j(\lambda, s)=j(\lambda, 0)=j(\lambda)$, and $R(\lambda, s)=R(\lambda, 0)=R_{0}^{0}$.

Let $\tau$ and $\sigma$ have positions $n$ and $n+1$, respectively, in the order $<$, and suppose that the claim is true of $\tau$ : Let $s_{0}$ be the least stage such that, for every $s \geq s_{0}$, $j(\tau, s)=j\left(\tau, s_{0}\right)=j(\tau)$ and $R(\tau, s)=R\left(\tau, s_{0}\right)=R(\tau)$. Then either $\tau$ never acts after $s_{0}$, in which case at $s_{0}+1$ we define the final values $j(\sigma)$ and $R(\sigma)$, or there is a least stage $s_{1}>s_{0}$ at which $\tau$ acts and ends the stage, in which case the final values $j(\sigma)$ and $R(\sigma)$ are defined at $s_{1}+1$.

The other claims about the mapping $\sigma \mapsto R(\sigma)$ are trivial by the definition of $R(\sigma, s)$.

Justified by the previous claim, we may define:

Definition 2.7. For every $\sigma$, a stage $t$ is $\sigma$-final if for every $s \geq t$, we have that $j(\sigma, s)=j(\sigma)$ and $R(\sigma, s)=R(\sigma)$.

Notice, that, by initialization, if $t$ is $\sigma$-final then we also have that for every $\rho$ with $\rho \leq \sigma$, and $s \geq t, j(\rho, s)=j(\rho)$ and $R(\rho, s)=R(\rho)$.

Claim 2. The functions $\sigma \mapsto j(\sigma), \sigma \mapsto R(\sigma)$, and $s \mapsto s_{\sigma}$ are $\Delta_{2}^{0}$.

Proof. Immediate by the Limit Lemma [14], since $j(\sigma, s)$ and $R(\sigma, s)$ are (extendible to) computable functions.

Claim 3. For every set $B$, and string $\sigma$,

$$
j(\sigma) \in \Theta_{\sigma}^{B} \Leftrightarrow \sigma^{=1} \cup\{|\sigma|\} \subseteq B .
$$


Proof. Let $s_{\sigma}$ be the least $\sigma$-final stage. If $\sigma^{=1} \cup\{|\sigma|\} \subseteq B$ then $j(\sigma) \in \Theta_{\sigma}^{B}$, by the validating axiom $\left\langle j(\sigma), \sigma^{=1} \cup\{|\sigma|\}\right\rangle \in \Theta_{\sigma}$. On the other hand, this is the only axiom for $j(\sigma)$ in $\Theta_{\sigma}$, as for every $\rho$ and $\tau$, the string $\tau$ can $\tau$-dump $j(\rho, s)$ into $\Theta_{\neq \tau}$ only if $\tau<\rho$ and acts at $s$, and thus no $\tau$ can $\tau$-dump $j(\sigma)$ after $s_{\sigma}$.

Claim 4. If $B \notin \Delta_{2}^{0}$ then there exists a strictly increasing sequence of prefixes of $B$,

$$
\sigma_{0} \subset \sigma_{1} \subset \cdots \sigma_{n} \cdots \subset B
$$

such that $R\left(\sigma_{n}\right) \neq R\left(\sigma_{n}^{+, B}\right)$, and for every requirement $R$ there exists $n$ such that $R\left(\sigma_{n}\right)=R$.

Proof. Suppose otherwise: By Claim 1 and the definition of the requirement assignment function, this amounts to assuming that there is $\sigma \subset B$ such that for every $\tau$,

$$
\sigma \subseteq \tau \subset B \Rightarrow R(\sigma)=R(\tau)
$$

We claim that in this case $B \in \Delta_{2}^{0}$. Fix such a $\sigma$ : We show how to use $\emptyset^{\prime}$ as an oracle to compute $B(n)$, by induction.

If $n<|\sigma|$ then $B(n)=\sigma(n)$.

Let now $n \geq|\sigma|$, and let $\tau=B \mid n$. With oracle $\emptyset^{\prime}$, compute $j(\tau)$, and the least $\rho$-final stage $s_{\rho}$, where $\rho$ is the string immediately following $\tau$ in the order $<$ of strings, and decide whether or not $j(\tau)$ is eventually realized (if so this must happens at $\left.s_{\rho}-1\right)$ :

- If so, then $B(n)=1$ (otherwise, the construction would make $R(\tau) \neq$ $\left.R\left(\tau^{+, B}\right)\right)$;

- if not, then $B(n)=0$ (otherwise, again, the construction would make $\left.R(\tau) \neq R\left(\tau^{+, B}\right)\right)$.

Claim 5. For every $B$ and $\sigma \subset B, j(\sigma) \in \Phi_{\sigma}^{\Theta_{\neq}^{B}}$ if and only if $j(\sigma)$ gets eventually realized.

Proof. Let $\sigma$ be given, and let $s_{\sigma}$ be a $\sigma$-final stage.

Assume that $j(\sigma) \in \Phi_{\sigma \neq \sigma}^{\Theta_{\neq \sigma}^{B}}$. Then there exists an axiom $\langle j(\sigma), F\rangle \in \Phi_{\sigma}$ such that $F \subseteq \Theta_{\neq \sigma}^{B}$. We claim that at any stage $s \geq s_{\sigma}$, this set $F$ is $\sigma$-accessible, i.e., if $\tau \leq \sigma$ and $j(\tau) \in F$, then $\tau^{=1} \cup\{|\tau|\} \subseteq \sigma^{=1}$. Clearly, $F \cap \omega^{[r(\sigma)]}=\emptyset$, and thus $j(\tau) \notin F$, if $r(\tau)=r(\sigma)$. So assume $r(\tau) \neq r(\sigma)$ (in particular $\tau \neq \sigma$ ), and let $\tau \leq \sigma$ : If $|\tau|<|\sigma|$ and $j(\tau) \in F$ then by Claim 3, in order to have $F \subseteq \Theta_{\neq \sigma}^{B}$ we must have $\tau^{=1} \cup\{|\tau|\} \subseteq B$, hence $\tau^{=1} \cup\{|\tau|\} \subseteq \sigma^{=1}$; if $|\tau|=|\sigma|$ (and $\tau \neq \sigma$ ), then there exists some $i \in \tau^{=1}-\sigma^{=1}$, giving $i \notin B$, thus by Claim $3 j(\tau) \notin F$, as $j(\tau) \notin \Theta_{\neq \sigma}^{B}$, since $j(\tau) \notin \Theta_{\tau}^{B}$. So at every stage $s \geq s_{\sigma}, F$ is $\sigma$-accessible, and $j(\sigma)$ is realizable, and thus has the opportunity of becoming realized, if not already realized.

In the other direction, suppose that $j(\sigma)$ is realized at some $s \geq s_{\sigma}$, through a suitable $\sigma$-accessible finite set $F$. By definition of a $\sigma$-accessible set, we have that for every $\tau \leq \sigma$, if $j(\tau) \in F$ then, as $\sigma^{=1} \subseteq B$,

$$
j(\tau) \in \Theta_{\neq \sigma}^{\sigma=1} \subseteq \Theta_{\neq \sigma}^{B} ;
$$

finally by $\sigma$-dumping all the other elements of $F$ into $\Theta_{\neq \sigma}$, the action of $\sigma$ permanently makes $F \subseteq \Theta_{\neq \sigma}^{B}$, yielding $j(\sigma) \in \Phi_{\sigma}^{\Theta_{\neq \sigma}^{B}}$. 
Claim 6. If $B \notin \Delta_{2}^{0}$, then the e-degrees of the sets $\Theta_{r}^{B}$ are computably independent.

Proof. Assume that $B \notin \Delta_{2}^{0}$. Let $\left\{\sigma_{n}: n \in \omega\right\}$ be a sequence of prefixes of $B$ as in Claim 4. We are now ready to show that for every requirement $R, B$ satisfies $R$. To this end, fix $R$, and let $n$ be such that $R\left(\sigma_{n}\right)=R$. We claim that

$$
B\left(j\left(\sigma_{n}\right)\right) \neq \Phi_{\sigma_{n}}^{\Theta_{\neq \sigma_{n}}^{B}}\left(j\left(\sigma_{n}\right)\right) .
$$

We distinguish the following two cases:

(1) $j\left(\sigma_{n}\right)$ never gets realized. In this case the following two properties hold, which together imply that $R$ is satisfied:

(a) $j\left(\sigma_{n}\right) \notin \Phi_{\sigma_{n}}^{\Theta_{\neq \sigma_{n}}^{B}}$ : This follows from Claim 5 .

(b) $j\left(\sigma_{n}\right) \in \Theta_{\sigma_{n}}^{B}$ : This follows from Claim 3, using that $\sigma_{n}^{=1} \cup\left\{\left|\sigma_{n}\right|\right\} \subseteq B$, as $B\left(\left|\sigma_{n}\right|\right)=1$ since $R\left(\sigma_{n}\right) \neq R\left(\sigma_{n}^{+, B}\right)$.

(2) $j\left(\sigma_{n}\right)$ eventually gets realized. The following hold:

(a) $j\left(\sigma_{n}\right) \in \Phi_{\sigma_{n}}^{\Theta_{\neq \sigma_{n}}^{B}}$ : This follows from Claim 5 .

(b) $j\left(\sigma_{n}\right) \notin \Theta_{\sigma_{n}}^{B}$ : This follows from Claim 3, using that $\sigma_{n}^{=1} \cup\left\{\left|\sigma_{n}\right|\right\} \nsubseteq B$, as $B\left(\left|\sigma_{n}\right|\right)=0$ since $R\left(\sigma_{n}\right) \neq R\left(\sigma_{n}^{+, B}\right)$.

This ends the proof of the claim.

We conclude this section with an open question. An s-operator is an e-operator $\Phi$ such that if $\langle x, D\rangle \in \Phi$ then $D=\emptyset$ or $D$ is a singleton. A set $A$ is said to be $s$-reducible to a set $B$ (denoted by $A \leq_{s} B$ ) if there exists an s-operator $\Phi$ such that $A=\Phi^{B}$ : It is easy to see that $\leq_{s}$ is reflexive and transitive, and thus one can consider the corresponding degree structure, where the $s$-degree of a set $A$ is the equivalence class of $A$ under the equivalence relation induced by $\leq_{s}$. The s-degrees form an upper semilattice with least element, which, as in the case of $\mathbf{0}_{e}$, consists of all the computably enumerable sets. (For more on s-degrees, and the importance of s-reducibility among the subreducibilities of $\leq_{e}$, see for instance [11].) Gutteridge's proof of the nonexistence of minimal e-degrees, and Lagemann's proof of Lemma 2.2, build in fact s-operators, and thus ipso facto yield results that hold of the s-degrees as well. On the contrary, in our construction, the enumeration of the validating axioms introduces e-operators that are not s-operators, and this seems to be necessary if we want to exclude from $\sigma$-accessible sets elements $j(\tau)$ for $\tau<\sigma$, but $|\tau|=|\sigma|$. So the following question arises naturally:

Question 2.8. Do there exist linearly ordered nontrivial initial segments of the s-degrees?

\section{Proof of Theorem 1.2}

We now show how to modify the proof of Theorem 1.1 to get Theorem 1.2. Given a set $A$, we define an $A$-e-operator to be a set $\Theta$ which is computably enumerable in $A$. Notice that if $\Theta$ is an $f$-e-operator, with $f$ a total function, then $\Theta^{B} \leq_{e}$ $f \oplus B$, as $\Theta \leq_{e} f$ by totality, being $\Theta$ computably enumerable in $f$. If a class $\left\{\Theta_{r}: r \in \omega\right\}$ of sets is computably enumerable in $f$ (i.e., the set $\left\{\langle x, r\rangle: x \in \Theta_{r}\right\}$ is computably enumerable in $f)$, then a similar argument shows that $\oplus_{r \in \omega} \Theta_{r}^{B} \leq_{e}$ $f \oplus B$; in particular if $f \leq_{e} B$ then $\left\{\operatorname{deg}_{e}\left(\oplus_{r \in \omega}\left(f \oplus \Theta_{r}^{B}\right)\right): r \in \omega\right\}$ is uniformly below $\operatorname{deg}_{e}(B)$. 
We need appropriate relativized versions of Lemma 2.2, and of the Main Lemma, given respectively by Lemma 3.1 and Lemma 3.2 below:

Lemma 3.1 (Lagemann [7]). If $f<_{e} B$, with $f$ a total function, and $B \in$ $\Delta_{2}^{f}$, then every countable partial order can be embedded in the degree interval $\left(\operatorname{deg}_{e}(f), \operatorname{deg}_{e}(B)\right)$.

Lemma 3.2 (Relativized Main Lemma). For every total function $f$, there is a family $\left\{\Theta_{r}: r \in \omega\right\}$ of $f$-e-operators such that for every $B$, if $B \notin \Delta_{2}^{f}$ then the set of e-degrees $\left\{\operatorname{deg}_{e}\left(f \oplus \Theta_{r}^{B}\right)\right\}$ is computably independent, and if $f \leq_{e} B$ then this set is also uniformly below $\operatorname{deg}_{e}(B)$.

Proof. Carry out the proof of the Main Lemma with the following provisos:

Modified requirements. The requirements now are:

$$
R_{i}^{r}: \quad\left(\forall B \notin \Delta_{2}^{f}\right)\left[\Theta_{r}^{B} \neq \Phi_{i}^{\oplus_{j \neq r}\left(f \oplus \Theta_{j}^{B}\right)}\right] .
$$

(Again, we build $\Theta_{j}$ so that for every $j, \Theta_{j}^{\omega} \subseteq \omega^{[j]}$, thus we may assume that $\bigoplus_{j \neq r}\left(f \oplus \Theta_{j}^{B}\right) \equiv_{e} f \oplus\left(\bigcup_{j \neq r} \Theta_{j}\right)^{B}$.)

Relativized Definition 2.5. Definition 2.5 should be modified as follows: A finite set $F$ is $\sigma$-accessible at $s+1$, if $F=H \oplus G$, with $H \subseteq f$, and $G \cap \omega^{[r(\sigma, s+1)]}=\emptyset$, and

$$
G \cap\{j(\tau, s+1): \tau \leq \sigma\} \subseteq\left\{j(\tau, s+1):|\tau|<|\sigma| \text { and } \tau^{=1} \cup\{|\tau|\} \subseteq \sigma^{=1}\right\} .
$$

As before, we say that $j(\sigma, s+1)$ is realizable at $s+1$ if there exists a $\sigma$-accessible finite set $F$, such that $j(\sigma, s+1) \in \Phi_{\sigma}^{s} F$.

Modified item (2) of Stage $s+1$ of the construction. In item (2) of Stage $s+1$ of the construction, if $F=H \oplus G$ is a suitable $\sigma$-accessible set, then $\sigma$-dump into $\Theta_{\neq \sigma}$ the relevant elements of $G$, i.e. the elements in $G-\{j(\tau, s+1): \tau \leq \sigma\}$ : This action makes $j(\sigma, s)$ realized.

The verification goes through without virtually any modification: Notice that it is decidable in $f$ whether a finite set $F$ is $\sigma$-accessible at a stage $s$, and thus whether $j(\sigma, s)$ is realized at $s$, hence the class $\left\{\Theta_{r}: r \in \omega\right\}$ is computably enumerable in $f$.

Proof of Theorem 1.2. Let $f \in \mathbf{a}$ and $B \in \mathbf{b}$, with $f<_{e} B$. Corresponding to $f$, let $\left\{\Theta_{r}: r \in \omega\right\}$ be the family of $f$-e-operators whose existence is ensured by the Relativized Main Lemma. Now, either $B \in \Delta_{2}^{f}$, in which case the claim follows from Lemma 3.1, or the e-degrees of the sets $f \oplus \Theta_{r}^{B}$ form a computably independent collection of degrees by Lemma 3.2, contained in the interval $(\mathbf{a}, \mathbf{b})$ and uniformly below $\mathbf{b}$, and thus we can apply Lemma 2.1 .

Theorem 1.2 shows that if $\mathbf{a}$ is total then in the initial segments of the principal filter $[\mathbf{a}, \infty)$ downwards density can always be accompanied by incomparability. Of course this does not hold of every principal filter, since it is known that density fails in the poset of the e-degrees: The density question for the e-degrees (raised by Rogers [12, p. 282]), was answered by Calhoun and Slaman [1], who exhibited a nontrivial empty open interval of e-degrees, in which both extremes of the interval 
contain $\Pi_{2}^{0}$ sets; more recently, Kent, Lewis, and Sorbi [6] have shown that strong minimal covers exist too.

Notice also:

Theorem 3.3 (Soskova and Soskov [16]). If $\mathbf{a}<\mathbf{b}$ are e-degrees and $\mathbf{b}$ contains a set $B$ which has a "special approximation" (which includes the cases of B total, or $B n$-cea, for $n \geq 2)$ then in the interval $(\mathbf{a}, \mathbf{b})$ one can embed every countable partial order.

Gutteridge [5] and Lagemann [7] showed that if $\mathbf{a}<\mathbf{b}$ and either $\mathbf{a}$ or $\mathbf{b}$ is total, then there exists $\mathbf{c}$, such that $\mathbf{a}<\mathbf{c}<\mathbf{b}$. We are able to show that also in this case density can be accompanied by incomparability:

Corollary 3.4. If $\mathbf{a}<\mathbf{b}$ and either $\mathbf{a}$ or $\mathbf{b}$ is total, then one can embed every countable partial order in the interval $(\mathbf{a}, \mathbf{b})$.

Proof. By Theorem 1.2 and Theorem 3.3.

\section{REFERENCES}

[1] W. C. Calhoun and T. A. Slaman. The $\Pi_{2}^{0}$ e-degrees are not dense. J. Symbolic Logic, 61:13641379, 1996.

[2] S. B. Cooper. Partial degrees and the density problem. J. Symbolic Logic, 47:854-859, 1982.

[3] S. B. Cooper. Enumeration reducibility, nondeterministic computations and relative computability of partial functions. In K. Ambos-Spies, G. Müller, and G. E. Sacks, editors, Recursion Theory Week, Oberwolfach 1989, volume 1432 of Lecture Notes in Mathematics, pages 57-110, Heidelberg, 1990. Springer Verlag.

[4] S. B. Cooper. Computability Theory. Chapman \& Hall/CRC Mathematics, Boca Raton, London, New York, Washington, DC, 2003.

[5] L. Gutteridge. Some Results on Enumeration Reducibility. PhD thesis, Simon Fraser University, 1971.

[6] T. F. Kent, A. E. M. Lewis, and A. Sorbi. Empty intervals in the enumeration degrees. Ann. Pure Appl. Logic, 163(5):567-574, 2012.

[7] J. Lagemann. Embedding Theorems in the Reducibility Ordering of the Partial Degrees. PhD thesis, M. I. T., 1971.

[8] D. Marsibilio and A. Sorbi. Bounded enumeration reducibility and its degree structure. Arch. Math. Logic, 51(1-2):163-186, 2012.

[9] K. McEvoy and S. B. Cooper. On minimal pairs of enumeration degrees. J. Symbolic Logic, 50:983-1001, 1985.

[10] P. Odifreddi. Classical Recursion Theory (Volume II), volume 143 of Studies in Logic and the Foundations of Mathematics. North-Holland, Amsterdam, 1999.

[11] R. Sh. Omanadze and A. Sorbi. Strong enumeration reducibilities. Arch. Math. Logic, 45(7):869-912, 2006.

[12] H. Rogers, Jr. Theory of Recursive Functions and Effective Computability. McGraw-Hill, New York, 1967.

[13] G. E. Sacks. Degrees of Unsolvability, 2nd Edition, volume 55 of Annals of Mathematical Studies. Princeton University Press, 1966.

[14] J. R. Shoenfield. On degrees of unsolvability. Ann. of Math., 69:644-653, 1959.

[15] R. I. Soare. Recursively Enumerable Sets and Degrees. Perspectives in Mathematical Logic, Omega Series. Springer Verlag, Heidelberg, 1987.

[16] M. I. Soskova and I. N. Soskov. Embedding countable partial orderings in the enumeration degrees and the $\omega$-enumeration degrees. J. Logic Computation, 2010.

Department of Mathematics, The University of California, Berkeley, 719 Evans Hall \#3840, Berkeley, CA 94720-3840 USA

Dipartimento di Scienze Matematiche e Informatiche "Roberto Magari", Universitá Degli Studi di Siena, Pian dei Mantellini , 44, I-53100 Siena, itAly 